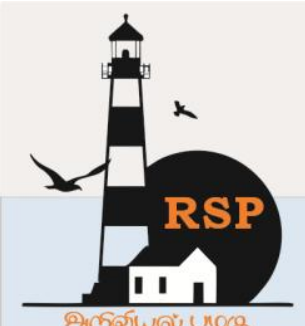

\title{
A Review on Recent Developments in Micro-channels for Cooling Applications
}

Jagannath Suresh ${ }^{1}$

${ }^{1}$ Dept. of Mechanical Engineering, National Institute of Technology Tiruchirappalli, Tamil Nadu, India jagannad101@gmail.com ${ }^{1}$

\begin{abstract}
Cooling of miniaturized spaces like electronic components is a major challenge to heat transfer engineers. Miniaturization of systems is an advantage and there has been a recent flourish of Micro Electro Mechanical Systems (MEMS). Hence, there is an obvious increase in demand for cooling of micro-devices and implementation of micro-channels is one of the most efficient ways for cooling applications in micro systems. The present paper discusses the applications of micro-channels, nature of flow in micro-channels, suitable materials which includes the recent developments in micro-channel material selection, fabrication aspects used for making micro-channels and the limitations which hinders a fully successful implementation of micro-channels. The use of fins and special materials recently introduced by the industry is also discussed.
\end{abstract}

Keywords: Micro-channel, Electronic components, Nano fluids, Heat transfer

\section{Introduction}

Micro-channels can be used for efficient cooling related to biological, chemical, and environmental applications. The size of the electronic parts like printed circuit boards is getting smaller in size, while their $\mathrm{I}^{2} \mathrm{R}$ heat generation is increasing. Devices like infrared detectors, superconductors, and laser mirrors are also incorporated with microchannels. Miniaturization is considered a boon by consumers and electronic engineers. But there is a tremendous challenge in the heat transfer aspects. The components should be maintained within the permissible temperature of approximately below $70^{\circ} \mathrm{C}$ to ensure the reliability of operations. Situations like change in temperature during start up, shut down, and load changes are to be taken care of. Besides the possibility of the formation of hotspots are also to be considered. It is expected that the heat flow of approximately $100 \mathrm{w} / \mathrm{cm}^{2}$ is expected in modern electronic components. In the present paper, various challenges related to microchannel cooling are summarised. Different arrangements in cooling and future challenges are Addressed.

\section{Nature of Flow in Micro-Channel}

As the channel size is getting reduced, the mean free path of molecules and the size of the channel becomes comparable. The nature of flow deviates from conventional continuum assumption. This is called a rarefaction effect. The flow becomes continuous when the Knudsen number is small. The large pressure drop makes considerable density changes and compressibility effects take a dominant effect. This factor is to be considered in design and modeling. The estimation of hydrodynamic and thermal entry length is important in various calculations. Surface roughness variation of even small magnitudes is important, as it can alter total flow values and change the regime of flows from laminar to turbulent.

\section{Use of Fins in Micro-Channels}

Different types of fins and grooves are tried in 
micro-channel. The heat transfer is changed by $12 \%(1)$. Vanapalli et al(2) tested a channel with nitrogen as fluid. This channel offered a very low friction factor. Various geometries like circles, squares, rhombuses, ellipses, etc.. in inline and staggered manner are tested. A special type of pillar is found to have the lowest friction factor. Fins will augment heat transfer by increasing area, provide a steeper velocity gradient. The length required for the development of the thermal boundary layer will be shorter.

\section{Fabrication of Micro-Channels}

A lot of techniques are available for the fabrication of micro-channels of suitable size. Photolithography and etching are some of the conventional methods typically used for the manufacturing of micro-channels. But their special nature limits the flexibility to form different shapes. One of the recent developments is the use of a 3D printed mold removal method to fabricate a micro-channel. The convenience of 3D printing methods and the use of PDMS materials encourage researchers to explore this mode of production. Several features like curved or rectangular serpentine and spiral paths are tried. (3). various shapes like sinusoidal 3D curved and cross-linked shapes are fabricated. It is also possible to modify the channel shape by modifying the orifice shape in the printer nozzle. Other methods like embossing, micro-milling processes, and laser ablation processing are also extensively used for the formation or manufacturing of micro-channels

The use of laser is a good option for microchannel fabrication (Molian et al 2009 (5)). The relative easiness, reduced time, and low cost involved make it attractive over some of the conventional processes. Damages during thermal aspects are a serious concern. It can be concluded that the minimum amount of burrs and redeposition, low fabrication time, the suitability of mass production are the advantages. The need for specially designed optics, beam delivery, and diagnostics, and high initial investment are the disadvantages. Some of the new techniques like micro milling offer a low energy consumption compared to other processes (Yu et al (6)).

\section{Materials}

Glass and silicon substrates are commonly used for the fabrication of micro-channels. Polymethyl methacrylate (PMMA)21 and polymethyl siloxane (PDMS) are some of the recently developed materials due to the low cost and possibility of disposable devices. PMMA is transparent and biocompatible and using for artificial drug delivery bio-implants etc. Materials like alloys, ceramics, and superalloys have been developed. For environments with high-temperature, metal aluminides offer a high degree of withstanding temperature effects. This may be attributed to the tendency of aluminides in aluminum to form protective oxide layers of aluminum oxides. Out of this Nickel aluminide (NiAl)can withstand very high temperatures. Polymers are relatively cheaper and offer properties like corrosion resistance. But this is associated with manufacturing difficulties. Metals offer good properties for manufacturing and use. But there are limitations like reactivity with fluids, lack of optical transparency. Hence this is not suitable for all applications. Silicon has high thermal conductivity and thermal-based manufacturing can cause harm in associated devices because of heat absorption. Ceramics and semiconductor devices are also tried extensively. But high cost restricts their uses.

\section{Limitations}

Some of the issues like manufacturing difficulty in polymers which are favored from a material point of view is a matter of concern. The high thermal conductivity of silicones also causes difficulty. Maskless photolithography process is good but depends upon the manufacturer's skill. Processes like Embossing and imprinting need the reproduction of micro features on a stamp. Eventually, these get worn out causing a change in channel dimensions

\section{Conclusions and Future Trends}

Increasing trends of miniaturization of components lead to restricted space availability is a major challenge in micro-channel manufacturing. Another concern is the increased heat generation due to a greater number of components like transistors. However, advances in manufacturing technology and research on newer compatible material can solve these problems to a great extent. Specialized fluids like nanofluids of suitable composition also help in addressing the challenges in micro-channel based cooling systems. Microchannels coupled with nanofluids is an emerging 
technique for systems like electronic components and other engineering devices.

\section{References}

\section{Journals}

[1] Solovitz, S. A., Computational Study of Grooved Microchannel Enhancements, Proceedings of ASME ICNMM200862128, Darmstadt, Germany, 2008.

[2] Vanapalli, S., ter Brake, H. J. M., Jansen, H. V., Burger, J. F., Holland, H. J., Veenstra, T. T., and Elwenspoek, M. C., Pressure Drop of Laminar Gas Flows in a Microchannel Containing Various Pillar Matrices, Journal of Micromechanics and Microengineering, vol. 17, pp. 1381-1386, 2007. [25] Lee, Y., Lee, $P$

[3] Welai.T, Hao.L, Liva.Z, Jiaping.S, Zongan.L, Nan.X, Jiguan.Y, Fabrication of different Microchannels by adjusting the Extrusion Parameters for Sacrificial Molds, Micromachines, 2019,10(8) 544

[4] Shashi Prakash and Subrata Kumar, Fabrication of microchannels a Review, Proceedings of Mechanical Engineers, 2015, Vol. 229(8) 1273-1288

[5] Molian P, Pecholt B, and Gupta S. Picosecond pulsed laser ablation and micromachining of $4 \mathrm{H}-\mathrm{SiC}$ wafers.

Appl Surf Sci 2009; 255: 4515-4520

[6] Yu H, Li B, and Zhang X. Flexible fabrication of three-dimensional multilayered microstructures using a scanning laser system. Sensor Actuat A: Phys 2006; 125: 553-564. 